\title{
EMPODERAMENTO E AUTONOMIA EM SAÚDE MENTAL: O Guia GAM como ferramenta de cuidado
}

\author{
EMPOWERMENT AND AUTONOMY IN MENTAL HEALTH: \\ guide the autonomous management of \\ medication as a tool
}

\section{Ana Carolina Machado Freitas', Juliana Beatriz Reckziegel', Rita de Cássia Barcellos²}

\section{RESUMO}

Objetivo: descrever a experiência de um grupo com usuários de um Centro de Atenção Psicossocial (CAPS) II, com a utilização do guia da Gestão Autônoma da Medicação como ferramenta de empoderamento e promoção da autonomia. Metodologia: Constitui-se recorte de um estudo de pesquisa e intervenção, consistindo a pesquisa ação qualitativa de cunho descritiva e compreensiva. Foram selecionados dez usuários, com nível mínimo de organização psíquica, vinculados ao CAPS II, indicados por profissionais de referência. Resultados: A partir da utilização do guia da Gestão Autônoma da Medicação identificou-se a relevância do grupo terapêutico, do vínculo, do território, da gestão do cuidado e autonomia dos sujeitos ao longo do tratamento, com vistas à melhoria da qualidade de vida e humanização das relações para usuários da saúde mental. Conclusão: o grupo pode permitir aos usuários do CAPS II tornarem-se mais críticos e autônomos em relação ao seu tratamento.

Descritores: Saúde Mental; Autonomia Pessoal; Grupo.

\begin{abstract}
Objective: To describe the experience of a group of users of a CAPS II, using the guide Medication Autonomous Management as an empowerment tool and promotion of autonomy. Methodology: It consists clipping a research study and intervention, consisting of a qualitative action research descriptive and comprehensive nature. Ten users were selected, with minimum level of psychic organization, linked to CAPS II, indicated by reference professionals. Results: From the use of the guide Autonomous Management of Medication identified the importance of the therapeutic group, the bond, planning, management of care and autonomy of the subjects throughout the treatment with a view to improving the quality of life and humanization of relations for users of mental health. Conclusion: the group allowed users CAPS II become more critical and autonomous in relation to its treatment.
\end{abstract}

${ }^{1}$ Especialista em Saúde Mental pela Universidade Federal de Santa Maria (UFSM), Santa Maria, RS, Brasil.

${ }^{2}$ Doutora em Educação pela Universidade del Mar (UDM), Viña del Mar, Chile.

Descriptors: Mental Health; Personal Autonomy; Group. 


\section{Introdução}

A utilização do Guia GAM, com base na tradução e adaptação do Guia de origem canadense (Quebec), voltado para atender as necessidades de pessoas em sofrimento mental grave, teve início por intermédio de um estudo multicêntrico, cujo objetivo era a elaboração do Guia Brasileiro da Gestão Autônoma da Medicação (Guia GAM-BR) e a sua consequente aplicação em Centros de Atenção Psicossocial (CAPS das cidades-piloto de Campinas-SP, Rio de Janeiro-RJ e Novo Hamburgo-RS. O Guia GAM, ofertado inicialmente nessas três cidades, consistiu-se de um conjunto de passos a serem propostos aos usuários com questões e informações destinadas a ajudá-lo a repensar as suas relações com o seu próprio cuidado e com o uso da de medicamentos psiquiátricos, objetivando ampliar o poder de negociação do sujeito com o seu próprio tratamento, junto à equipe que o acompanha e junto aos demais membros da esfera sócio-afetiva do sujeito ${ }^{1}$. As questões propostas pelo guia vão desde o simples falar sobre as suas próprias expectativas quanto à gestão autônoma de sua medicação, ouvir e ser ouvido até, por exemplo, se dar conta de que ele é uma pessoa e não uma doença.

Este artigo decorre do processo de formação em Programa de Residência Multiprofissional Integrada em Saúde Mental, em nível de pós-graduação, em um município do interior do RS. O GAM emerge como uma iniciativa que visa estimular aos profissionais dos serviços de saúde mental desse município interiorano para a utilização do referido Guia, uma vez que o seu manejo é de extrema facilidade bem como à aplicação do mesmo. Ressalta-se que o guia é uma ferramenta potencializadora na construção de estratégias para a produção de saúde no cuidado de usuários advindos dos serviços de saúde mental. Além disso, o Guia GAM se mostra como uma estratégia através da qual os usuários aprendem a cuidar do uso dos medicamentos, considerando seus efeitos em todos os aspectos da vida. O mesmo seria a ferramenta que, quando bem conduzida, levaria o usuário de saúde mental a gestão autônoma da medicação de forma a reorganizar a sua vida cotidiana efetivando o tratamento².

A relevância desta pesquisa sustentou-se a partir da observação empírica de que os usuários do serviço de saúde mental fazem uso de altas doses de psicofármacos, sem que ao menos possuíssem qualquer ideia "do que" e "por que" consumiam aqueles medicamentos. A partir da referida situação e da necessidade de buscar resolutividade para tal demanda, optou-se por implantar o grupo do guia Gestão Autônoma da Medicação e acompanhar o seu desenvolvimento em um CAPS II.

Atualmente, entende-se que o processo de adoecimento mental advém de um conjunto de fatores sociais, biológicos, econômicos e psicológicos, afetando todos os segmentos da população, acarretando prejuízos e sofrimento para os indivíduos, família e sociedade. Os dados de pessoas que sofrem com transtornos mentais têm aumentado (cerca de 450 milhões de pessoas no mundo), mas apenas uma pequena parcela recebe tratamento adequado ${ }^{3}$.

0 cuidado em saúde mental requer uma maior atenção, pois o adoecimento psíquico é significativo e tem se mostrado bastante relevante. Quase $20 \%$ da população do país necessitam de algum cuidado na área de saúde mental e $3 \%$ apresentam transtornos mentais severos e persistentes necessitando de cuidados contínuos ${ }^{4}$. Os medicamentos destinados ao tratamento das doenças mentais ocupavam, em 1985, o $9^{\circ}$ lugar entre os mais vendidos mundialmente, representando $6,9 \%$ das vendas 5 .

O Movimento da Reforma Psiquiátrica propôs um novo modelo de atenção à saúde mental, o qual passa a considerar o usuário em sua subjetividade, alem de instituir uma nova política de saúde mental, criando os CAPS para o tratamento dos usuários. Os CAPS, além das estratégias medicamentosas e psicoterapêuticas, utilizam ações de lazer e de cultura como formas legítimas e eficazes na produção de vida e de saúde dos usuários. Mesmo após o movimento da Reforma Psiquiátrica, o tratamento continua baseando-se no uso excessivo de medicamentos, com esclarecimentos incipientes ais usuários de saúde mental sobre sua utilização e efeitos.

Essa pesquisa, portanto se deve ao fato de que a equipe de residentes de um CAPS tipo II do município de Santa Maria, viu-se diante do desafio de ser cooresponsável pela implantação desse dispositivo naquele serviço. Nesse sentido, o processo de utilização do guia da Gestão Autônoma da Medicação, poderá oportunizar o acompanhamento desses usuários, agindo como um instrumento de informação de seus direitos em relação ao seu tratamento e possivelmente proporcionar um espaço de construção de autonomia e de gestão do autocuidado.

Em síntese, o objetivo desse artigo pautou-se na descrição da experiência decorrente do processo de atendimento grupal com usuários de um CAPS II, utilizando o guia da gestão autônoma da medicação como tecnologia leve de cuidado em saúde. 0 processo de desenvolvimento do grupo possibilitou a constituição do mesmo como um grupo terapêutico, oportunizando a escuta, o vínculo, a ocupação do território e estimulando a reinserção social, autonomia e empoderamento para ampliar o autocuidado, melhorando assim a qualidade de vida dos usuários de saúde mental. 


\section{Metodologia}

Este estudo é parte de um projeto de pesquisa e intervenção (projeto guarda-chuva), o qual passou pela aprovação do Comitê de Ética e Pesquisa, sob Certificado de Apreciação e Aprovação Ética (CAAE) 46053615.2.0000 5346, parecer aprovado em 04/08/2015, vinculado ao programa de Residência Multiprofissional Integrada em Saúde Mental, o qual foi executado em nível de atenção secundário, sendo CAPS II.

Trata-se de uma pesquisa ação de cunho descritiva e compreensiva:

Toda pesquisa-ação é considerada participativa sendo necessário o envolvimento das pessoas implicadas nos problemas e, somente é considerada como tal, se houver uma ação por parte do grupo de pessoas sob observação. Essa ação por sua vez, deverá ter um caráter prático e não apenas prosaico ${ }^{6}$.

O autor propõe a assertiva de que a pesquisa ação parte do pressuposto de que os indivíduos envolvidos nesse procedimento compõem um grupo com objetivos e metas em comum. Dessa forma a ação estaria vinculada ao modo de ser e seria possível identificar o que é no que se faz, considerando que essas ações são práticas sociais, as quais constituíssem a partir da historicidade de cada sujeito ${ }^{7}$.

No que concerne ao desenho investigativo, à intervenção se deu a partir da constituição de um grupo que utilizou o Guia da Gestão Autônoma da Medicação como ferramenta mediadora e contou com a participação de usuários do CAPS II, indicados por profissionais de referências. O funcionamento do grupo compreendeu o período de abril a novembro do ano de 2015, totalizando vinte e quatro encontros, realizados uma vez por semana, com duração aproximada de 1h15min cada encontro. Participaram inicialmente dez usuários portadores de transtornos mentais graves em tratamento no serviço CAPS II. Estes foram selecionados obedecendo alguns critérios de inclusão. No momento do convite para participar do grupo, os usuários deveriam apresentar-se com um nível mínimo de organização psíquica, ou seja, deveriam apresentar condições de desenvolver o diálogo, capacidade de compreensão e de certa interação social para que fossem capazes de fornecer respostas ao guia, além de participarem dos encontros e desenvolverem as tarefas propostas. Ao final dos encontros, dos dez participantes selecionados somente cinco concluíram o processo investigativo, isto ocorreu devido a desestabilizações sofridas pelo usuário no âmbito de sua doença mental, fazendo com que o mesmo não apresentasse mais as condições necessárias para dar andamento a sua participação no grupo. A pesquisa foi conduzida de acordo com os padrões éticos exigidos.

Durante o desenvolvimento do grupo, os profissionais pesquisadores descreveram em diário de campo as principais impressões do funcionamento do grupo. O objetivo do diário de campo foi captar falas dos sujeitos, histórias de vida, emoções, depoimentos, sentimentos e comportamentos.

Propõe-se que o diário de campo se caracterize por anotações onde o investigador descreve o que identifica. Tais anotações são impressões pessoais que podem se modificar com o desenvolvimento do grupo, conversas informais, observações de comportamento, interferências dos interlocutores ${ }^{8}$. Os diálogos serão transcritos na íntegra, sem correção das normas culta da língua portuguesa, trazendo a fala dos usuários no decorrer do grupo.

Os pesquisadores tomaram o cuidado de estarem sobre constante supervisão e orientação para evitar possíveis interferências nas respostas obtidas com usuários participantes do Grupo GAM. Os usuários aceitaram participar da pesquisa, após concordarem e assinarem o Termo de Consentimento Livre e Esclarecido (TCLE), orientados sobre o objeto e os objetivos da pesquisa. Após o término do grupo, realizou-se a análise do material coletado por meio de saturação de dados. Conforme o autor,

A amostragem por saturação é uma ferramenta conceitual frequentemente empregada nos relatórios de investigações qualitativas em diferentes áreas no campo da Saúde. É usada para estabelecer ou fechar o tamanho final de uma amostra em estudo, interrompendo a captação de novos componentes. Noutras palavras, as informações fornecidas pelos novos participantes da pesquisa pouco acrescentariam ao material já obtido, não mais contribuindo significativamente para o aperfeiçoamento da reflexão teórica fundamentada nos dados que estão sendo coletados ${ }^{9}$. 


\section{Resultados e discussão}

A discussão do resultado foi apresentada em três momentos. Primeiramente, discutiu-se a relevância das tecnologias em saúde, enfatizando a tecnologia leve de cuidado como fundamental na constituição do Grupo GAM. Na sequência, foram exploradas as estratégias do Grupo GAM junto aos usuários, finalizando com a descrição do funcionamento do grupo, assim como a apresentação de verbetes ou expressões trazidas pelos usuários, os quais caracterizam a "ciranda de palavras", sugerindo o quanto estas expressões orbitam e circulam no cotidiano dos sujeitos.

\section{O Grupo GAM como tecnologia leve de cuidado}

As ferramentas que contribuem para o cuidado e produção de saúde são diversas e vem sendo cada vez mais exploradas. Tem-se como objetivo o aperfeiçoamento da assistência integral e resolutividade no gerenciamento do processo saúde - doença da população. Essas tecnologias são definidas com leves, leves-duras e duras.

As tecnologias em saúde são divididas em leves, leve-duras e duras. Às leves compreendem as relações interpessoais, como a produção de vínculos, autonomização e acolhimento; as leve-duras dizem respeito aos saberes bem estruturados, como a clínica médica, a epidemiologia e a clínica psicanalítica; e as duras são compostas por equipamentos tecnológicos do tipo máquina, normas e estruturas organizacionais ${ }^{10}$.

Essas tecnologias agregam valor à reflexão a cerca do cuidado com a finalidade de proporcionar integralidade em saúde, tornando as tecnologias leves fundamentais para a compreensão da subjetividade dos usuários em saúde mental. Sugere que:

Podemos enfatizar que a organização das práticas de saúde e das relações terapêuticas na produção do cuidado com ênfase nas tecnologias leves possibilita de forma efetiva e criativa de manifestação de subjetividade do outro, a partir dos dispositivos de acolhimento, vínculo, autonomia e responsabilização contidos nessa organização da assistência á saúde ${ }^{11}$.

Nesse contexto, podemos citar como exemplo de tecnologia leve usada recentemente em saúde mental, o guia da Gestão Autônoma da Medicação. O mesmo surgiu como proposta na gestão do uso de psicofármacos que se iniciou em Quebec/Canadá, na década de 1990, emergindo a partir da problematização realizada por movimentos sociais acerca do uso de psicofármacos ${ }^{2}$. Voltado a usuários com transtornos mentais graves, tornou visível a pluralidade de posições em face da medicação, reconhecendo o direito ao consentimento livre e esclarecido para utilização de psicofármacos e a necessidade de compartilhar as decisões entre profissionais e usuários ${ }^{12}$.

O guia da Gestão Autônoma da Medicação adaptado para o Brasil possui cinco passos, nos quais os usuários são orientados a pensarem como os psicofármacos foram introduzidos em suas vidas, quais são seus direitos na saúde mental, relatos de suas experiências com o uso de outros medicamentos bem como 0 acesso aos mesmos. $O$ alinhamento da discussão desses temas está na relevância das experiências de vidas dos usuários, ressaltando o empoderamento para lidar com os psicofármacos que fazem uso, sempre contando com suporte da equipe de saúde e rede social².

\section{grupo GAM e suas implicações com usuários de saúde mental}

O espaço territorial ocupado para realização do grupo da Gestão Autônoma da Medicação foi a Biblioteca Municipal. Além de seu papel fundamental de promoção de reinserção social, provocou nos usuários sentimentos de pertencimento social, no momento em que paralelo o desenvolver do grupo realizaram outras atividades como: a leitura de jornais, revistas e livros disponíveis no espaço da biblioteca municipal; Deslocamento ao centro da cidade para participação no grupo ao invés da tradicional "ida ao CAPS". O sentar-se na praça em frente à biblioteca para conversas com os colegas; Aproximação com os profissionais residentes envolvidos durante os encontros; Contato com funcionários e outras pessoas que circulam no espaço da biblioteca municipal e confraternizações envolvendo todo o Grupo GAM, após período de férias e feriados. Tais estratégias foram estimuladas pelos profissionais residentes que, agregadas 
ao trabalho desenvolvido no grupo da Gestão Autônoma da Medicação, reforçaram o processo de empoderamento e autonomia aos usuários do CAPS II, estimulando a pro atividade dos mesmos. A autonomia pode ser definida como:

Ação autônoma que pressupõe a liberdade de ação requer que a pessoa seja capaz de agir conforme suas escolhas e decisões tomadas. Uma pessoa autônoma é aquela que tem liberdade de pensamento e é livre para escolher as opções que lhe são apresentadas ${ }^{13}$.

Agregado ao conceito de autonomia aparece o termo empoderamento, tido como:

Fortalecimento de poder, participação e organização dos usuários e familiares no âmbito da produção de cuidado em serviços formais ${ }^{14}$.

No desenvolver do grupo da Gestão Autônoma da Medicação, usuários trouxeram em seu discurso temas que se mostraram significativo e relevante, sendo em sua maior parte relacionados com questões de preconceito, estigma, solidão e medo vivenciados em seus cotidianos.

A discriminação assume muitas formas, afeta diversas áreas fundamentais da vida e (quer de maneira aberta ou involuntária) é generalizada. A discriminação pode influir no acesso de uma pessoa a tratamento e atenção adequados, bem como em outras áreas da vida, como emprego, educação e abrigo. A incapacidade de integrar-se devidamente à sociedade como consequência dessas limitações pode aumentar o isolamento experimentado pelo indivíduo, o que pode, por sua vez, exacerbar o transtorno menta/15.

Depreende-se então que os usuários com adoecimento mental podem estar mais propensos a situações de vulnerabilidade e de violência e dolo de deus direitos, pois, em algumas situações e em determinado tempo, estão impossibilitados de responder por suas necessidades e tratamento, ficando desprotegidos.

\section{Empoderamento e autonomia: pistas importantes para a saúde mental}

Durante a experiência com a utilização do Guia GAM, identificou-se a viabilidade do processo grupal terapêutico, intensificando a técnica da escuta, fortalecendo o vínculo entre usuário-profissional reforçando, portanto as relações interpessoais entre os participantes do grupo, profissionais e sociedade. A formação do vínculo ocorre com a aproximação entre trabalhador de saúde e usuário, ambos com suas intenções, necessidades e sentimentos, mas em situações diferentes, uma vez que o usuário, com estado emocional ou físico fragilizado, busca assistência junto ao outro, profissional supostamente capacitado para cuidar da causa de sua fragilidade ${ }^{16}$.

O vínculo com usuários ampliou a eficácia das ações de saúde, sob certos aspectos favorece a participação do usuário frente a seu tratamento, não sendo possível se estabelecer vínculo sem que o usuário seja reconhecido como sujeito que deseja, fala e é capaz de julgamentos ${ }^{17}$. 0 vínculo ainda envolve afetividade e respeito, estimulando a cidadania e autonomia do usuário ${ }^{18}$.

O grupo terapêutico mostrou-se como uma ferramenta facilitadora para estreitar os vínculos entre usuários e profissionais, promovendo as trocas de experiências e histórias de vida entre os usuários da saúde mental, surgindo como espaço para resolutividade, se consideradas as pequenas estratégias e iniciativas que auxiliaram no gerenciamento do tratamento, relações familiares, ocupação de equipamentos sociais, inclusão social e de outras questões que envolvem o tratamento e suas vidas. Um grupo terapêutico permite o compartilhamento de experiências, trocas dialógicas e melhoria na adaptação do modo de vida tanto individual quanto coletivamente ${ }^{19}$. A finalidade de um grupo psicoterapêutico é a possibilidade de socialização da palavra através das falas individuais e da escuta do outro, construindo sentido tanto para o indivíduo quanto para o grupo, fatores que favorecem o autoconhecimento ${ }^{20}$.

A experiência com a constituição e implantação do Grupo GAM em um CAPS II, tem se mostrado positiva, devido ao fortalecimento da gestão e autonomia do cuidado para o usuário com transtorno mental. A gestão autonomia da medicação deve considerar os usuários como protagonistas e cooresponsáveis na gestão de seu tratamento, participando das decisões relacionadas ao uso de suas medicações. A autonomia não se constrói de maneira solitária, mas na relação com o outro, está atrelada ao fato de criar e manter laços entre as pessoas ${ }^{12}$. 
O Grupo GAM teve como proposta fomentar e estimular o empoderamento e autonomia dos usuários de saúde mental, fazendo-os mais críticos em relação ao tratamento e ao uso de medicamentos psicotrópicos, vindo ao encontro do objeto de pesquisa e intervenção dos profissionais residentes. Optou-se por desenvolvê-lo com a ocupação de um dispositivo social no território, a Biblioteca Municipal. Este dispositivo foi escolhido seguindo o referencial da política de reforma psiquiátrica a qual privilegia a utilização de espaços sociais como produtores de saúde, fortalecendo o movimento de reinserção dos usuários de saúde mental em espaços de base territorial, promovendo a inclusão social desse público, podendo assim, protagonizar o seu próprio cuidado.

A reinserção social pode ser entendida como a reconstrução das perdas e o fortalecimento ou criação de uma rede de apoio, resgate ou estabelecimento de uma rede social comprometida ou inexistente. $O$ objetivo da reinserção social é a capacitação do indivíduo para exercer o seu direito à cidadania, implicando em uma revisão dos estigmas sociais estabelecidos ${ }^{21}$. É essencial que os CAPS sejam capazes de desenvolver relações com os vários recursos existentes no âmbito de sua comunidade ${ }^{22}$. Trata-se de produzir territórios existenciais que possibilitem reinventar a vida em seus aspectos cotidianos ${ }^{23}$.

Além das experiências vivenciadas e trazidas pelos usuários no decorrer do grupo, vieram à tona falas contendo estratégias e iniciativas desenvolvidas por eles, mostrando o reflexo da vida cotidiana em cada um e suas capacidades para amenizar o sofrimento de conviver com a doença mental. Tais falas podem ser vistas nos seguintes trechos: "Faço varias coisas para me cuidar da minha saúde e melhorar, verifico pressão, cuido da alimentação e faço caminhada..."; "Saio, caminho, limpo e vejo TV e converso. Leio revista e jornal. Faço grupos no CAPS. Não bebo bebida de álcool".

Diante desse discurso, identificou-se a relevância do espaço de interação social para os usuários, sendo que o fato deles conseguirem circular em outros ambientes diversos de sua casa e do CAPS os torna mais capazes de enfrentar e lidar com o adoecimento mental. Além disso, o grupo tornou-se um espaço que possibilitou aos usuários a manifestação de sentimentos até então não ditos ou expressados, como problemas familiares, dificuldades de falar devido à exposição, superação da timidez, entrosamento com o outro e empatia com as histórias de vida dos membros do grupo, sentimentos característicos de pertencimento a um grupo.

Corroborando com as ideias expressas acima, tornou-se possível pressupor que o Grupo GAM foi um espaço de amadurecimento, trocas e aprendizagem em relação aos usuários, ficando claro no encerramento desse processo que os usuários conseguiram adquirir um maior grau de empoderamento, aproximando-se de seu tratamento e com mais autonomia em suas relações, como exemplificado no relato a seguir: "Posso ter direito de buscar ajuda para participar do meu tratamento. Participar de grupos, oficinas, porque é pra sempre."; "Foi bom participar desse grupo, porém tem que se cuidar com os efeitos da medicação".

Com o encerramento do grupo, os usuários trouxeram espontaneamente depoimentos que mostram como as ações desenvolvidas acrescentaram positivamente no tratamento de cada um, assim como, uma experiência significativa que o grupo deixou em suas vidas.

\section{Ciranda de expressões grupais}

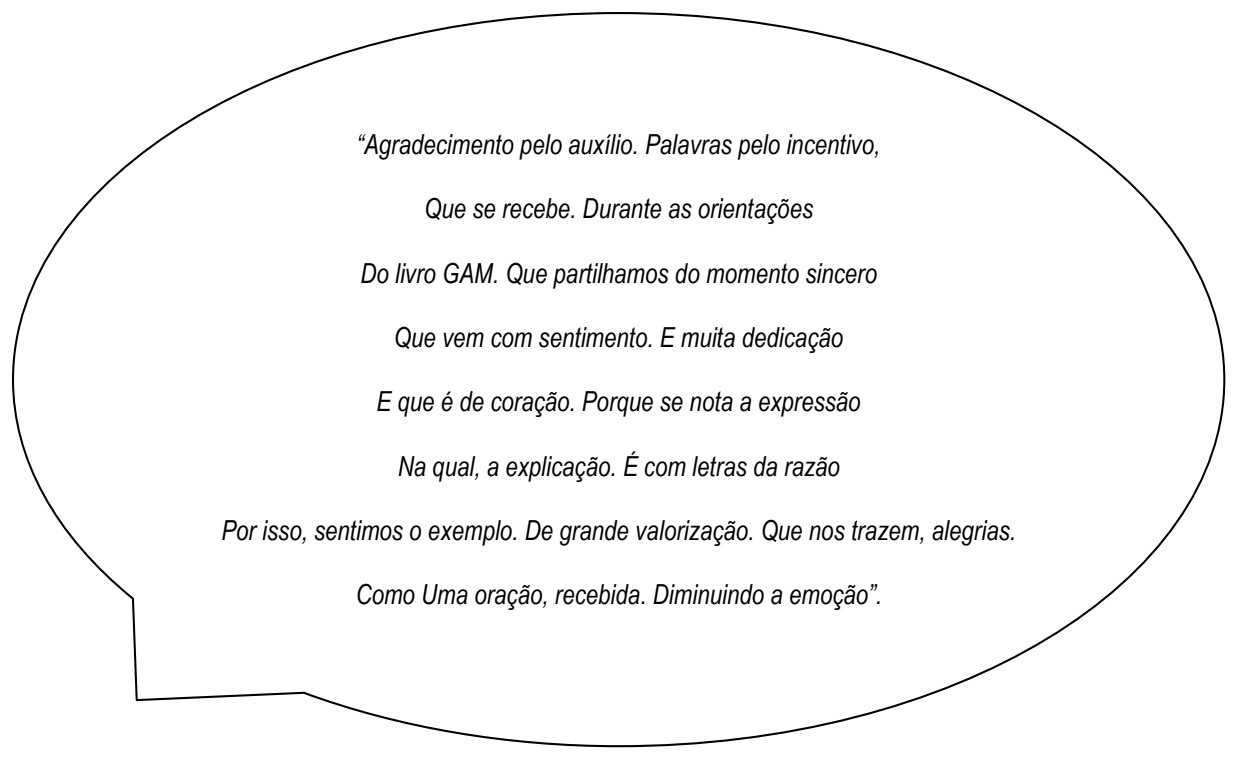


Concluindo esta etapa analítica, fica marcado mais um relato de um dos usuários participantes: "As pessoas não aceitam a minha doença, são indiferentes porque acham que não sou "normal", não tenho direito de opinar. Queria que tivessem interesse em mim".

\section{Considerações Finais}

É possível concluir, deste modo, que o grande desafio instaurado para os profissionais e familiares vinculados no cuidado de usuários com transtornos mentais graves é a sensibilização e prática da humanização das relações interpessoais. Uma vez que se considere o usuário como sujeito de direitos, capaz, produtivo e útil na sociedade, tornamse visível suas potencialidades, indo além do adoecimento mental e contribuindo para o sucesso do tratamento, assim como, a melhoria da qualidade de vida dos mesmos. A atividade desenvolvida por meio do grupo teve como ferramenta mediadora o Guia GAM, uma tecnologia leve e inovadora na assistência a saúde dos usuários de saúde mental, aliada a ocupação de espaços públicos, construção de espaço terapêutico, apoio para desenvolvimento de estratégias de enfrentamento da doença mental, e estímulo para desenvolvimento de relações interpessoais. Sendo assim, entende-se que o presente trabalho foi significativo e refletiu no autocuidado e ampliação das interações sociais, fazendo com que os usuários participantes se tornassem mais críticos em relação a seus direitos e deveres, colaborando para a qualidade de vida e qualificação do tratamento em saúde mental.

\section{Referências}

1. PASSOS E. et al. Autonomia e co-gestão na prática de saúde mental: 0 dispositivo da gestão autônoma da medicação (GAM). Aletheia 41, p.24-38, maio/ago. 2013.

2. SANTOS. D.V.D. A Gestão Autônoma da Medicação: Da prescrição à escuta. Campinas. São Paulo, 2014.

3. ORGANIZAÇÃO MUNDIAL DA SAÚDE. Relatório Sobre A Saúde No Mundo. Saúde Mental: Nova Concepção.Nova Esperança. 2001.

4. BRASIL. Ministério da Sáude. Secretaria Executiva. Coordenação Nacional de DST/Aids. A politica do Ministério da Saúde para a Atenção Integral a Usuários de Álcool e Outras Drogas. Brasília. 2003.

5. ALMEIDA.et.al. Consumo de psicofármacos em uma região administrativa do Rio de Janeiro: a llha do Governador. Cad. Saúde Pública vol.10 n.1 Rio de Janeiro Jan./Mar. 1994.

6. THIOLLENT. M. Metodologia da pesquisa-ação. 14ª ed. São Paulo (SP): Cortez, 2005.

7. PIMENTA, S.G. Pesquisa-ação crítico colaborativa: construindo seu significado a partir de experiências com a formação de docentes. Educação e Pesquisa [periódico na Internet]. 2005 Set-Dez [acesso em 29 de outubro de 2015]; 3(3):52139. Disponível em: http:// www.scielo.br/pdf/ep/v31n3/a13v31n3.pdf

8. MINAYO, M.C.S. O desafio do conhecimento: Pesquisa qualitativa em saúde - 14ed. São Paulo: Hucitec, 2014.

9. FONTANELLA, B.J.B. Janete Rica. Egberto Ribeiro Turato. Amostragem por saturação em pesquisas qualitativas em saúde: contribuições teóricas. Cad.Saúde Pública, Rio de Janeiro, 24(1):17-27, jan, 2008.

10. MENDES-GOLÇALVES, R.B. Tecnologia e organização social das práticas de saúde. São Paulo: Hucitec; 1994.

11. AYRES, J.R.C.M. Cuidado e reconstrução das práticas em saúde. Interface. 2004; 8 (14);73-92.

12. CAMPOS, et.al. GUIA DA GESTÃO AUTÔNOMA DA MEDICAÇÃO - GAM -DSC/FCM/UNICAMP; AFLORE IPUB/ UFRJ; DP/UFF; DPP/UFRGS, 2012.

13. COSTA, J.R.E et al. Para compreender a doença numa perspectiva de bioética. Centro Universitario São Camilo. 1 (2);103-110, 2007.

14. VASCONCELOS. E.M. Abordagens psicossociais. Reforma Psiquiatrica e Saúde Mental na Otica da Cultura e Lutas populares. SP. Hucitec, 2008.

15. OMS. Livro de recursos da OMS sobre saúde Mental, direitos humanos e legislação. Genebra. 2005.

16. BRASIL. Ministério da Saúde. Humaniza SUS: Política Nacional de Humanização: Documento Base para Gestores do SUS. Brasília. 2004.

17. SCHIMIDT, M.D. LIMA.M.A.D.S. Acolhimento e vínculo em uma equipe do Programa de Saúde da Família. Cad. Saúde Pública. 2004; 20(6). 
18. MACHADO, M.F.A.S. Compreensão das mudanças comportamentais do usuário no PSS por meio da participação habilitadora (tese). Fortaleza: Faculdade de Farmácia, Odontologia e Enfermagem, Universidade Federal do Ceará; 2007. 19. BENEVIDES, D.S. et al. Cuidado em saúde mental por meio de grupos p p psicoterapêuticos de um hospital dia: Perspectivas dos trabalhadores de saúde. Interface - Comunic., Saúde, Educ., v.14, n.32, p.127- 38, jan./mar. 2010. 20. PITTA, A.M.F. Reorientação do modelo de Atenção: Equidade e Justiça Social na Organização de Serviços de Saúde Mental. In: BRASIL. Ministério da Saúde. Conselho Nacional de Saúde Caderno de Textos de Apoio da III Conferência Nacional de Saúde Mental. Brasília, Ministério da Saúde / Conselho Nacional de Saúde, 2001. Disponível em <http:// bvsms.saude.gov.br/bvs/publicacoes/3conf_mental.pdf>

21. OBID. Observatório Brasileiro de Informações sobre Drogas. Reinserção Social: definição. Brasília, 2007.

22. AMARANTE. P. Loucos pela vida: a trajetória da reforma psiquiátrica no Brasil. Rio de Janeiro. Editora: Fiocruz, 1995.

23. RAUTER,C. Oficinas pra que? Uma proposta ético-estético-política para oficinas terapêuticas. In. AMARANTE, P. (org). Ensaios: subjetividades, saúde mental, sociedade. Rio de Janeiro. Fiocruz. 2000.

\section{Ana Carolina Machado Freitas}

Endereço para correspondência - Rua: Ernesto Barros, n¹345, Bairro: Santo Antonio, CEP: 96506-322, Cachoeira do Sul, RS, Brasil.

E-mail: carolpsicofreitas@gmail.com Lattes: http://lattes.cnpq.br/3950149480193042

Juliana Beatriz Reckziegel - Juliana.reckziegel4@gmail.com

Rita de Cássia Barcellos - dra.ritabarcellos@gmail.com

\section{Enviado em 26 de janeiro de 2016. Aceito em 20 de junho de 2016.}

